\title{
note
}

\section{Les conditions d'utilisation d'un lecteur de dosimètres TL à chauffage traditionnel et d'un lecteur automatique à chauffage rapide. Leur comparaison *}

\author{
G. MARINELLO **, J. POLLACK ** \\ et $P$. BLANCHARD ${ }^{* * *}$, J. BARTHE ***
}

(Manuscrit reçu le 11 mai 1989)

RÉSUMÉ Les auteurs comparent les propriétés dosimétriques du borate de lithium dopé au manganèse ou au cuivre, selon qu'il est lu sur un lecteur à chauffage traditionnel ou sur un nouveau type de lecteur automatique à chauffage rapide. Lorsque les conditions de réglage du préchauffage et du chauffage sont optimales, le fading thermique est indépendant du type de lecteur et la reproductibilité des mesures est très bonne. De même, la courbe de réponse en fonction de la dose ne dépend que du matériau radiothermoluminescent (RTL) et non du type de lecteur, sous réserve qu'elle soit normalisée à 1 pour une même dose. Par contre, la réponse en fonction de l'énergie dépend du mode de chauffage. Enfín, une propriété particulière du borate de lithium dopé au cuivre est mise en évidence : sa réponse n'est pas proportionnelle à la masse du matériau RTL. L'exploitation judicieuse de cette dernière propriété permet d'éviter la pesée des échantillons de poudre avant toute mesure et donc de gagner un temps considérable.

ABSTRACT The dosimetric properties of lithium borate activated with manganese or copper have been compared according to the heating procedures, i.e. with either a traditional heating reader or a new fast heating device associated with an automatic reader. When the adjustements of preheating and main heating are made optimal, fading becomes independent of the reader used and the reproducibility of the measurements is very good. The response curve of the TL material vs the dose is also found to be independent of the reader, provided it is normalized to 1 for the same reference dose. On the other hand the variation of the response vs energy (photon or electron beams) depends upon the heating procedures. Finally a particular characteristic of $\mathrm{Li}_{2} \mathrm{~B}_{4} \mathrm{O}_{7}: \mathrm{Cu}$ has been demonstrated : the response corresponding to a given dose is not proportional to the mass of the TL material used for the reading. As an interesting consequence it is possible to avoid weighing each reading sample and therefore to save time.

* Communication présentée lors du Séminaire sur la dosimétrie in vivo en radiothérapie et en radiodiagnostic, Nice, 23-24 février 1989.

** Unité de Radiophysique, Service de Carcinologie de l'hôpital Henri Mondor, 51, avenue du Maréchal-de-Lattre-de-Tassigny, 94010 Créteil.

*** Commissariat à l'énergie atomique, IPSN, Département de protection technique, DPT/ SIDR, BP $n^{\circ} 6,92230$ Fontenay-aux-Roses. 
Largement utilisée en radioprotection, la dosimétrie par radiothermoluminescence (RTL) l'est aussi, de plus en plus, dans le domaine médical. Mais elle est délicate à pratiquer et souvent longue à mettre en œuvre, en particulier si on ne dispose que d'un lecteur manuel à chauffage traditionnel. Un nouveau type d'appareil de lecture mis au point par G. PORTAL et son équipe [10] permet un gain de temps important sous réserve que le matériau associé soit judicieusement choisi. C'est ce que nous allons essayer de montrer, tout en étudiant les propriétés dosimétriques de deux matériaux RTL équivalents au tissu largement utilisés pour les applications médicales, le borate de lithium dopé au manganèse ou au cuivre.

\section{MATÉRIEL ET MÉTHODE}

\subsection{Les deux types de lecteurs}

- Le lecteur SAPHYMO STEL LDT 22 est un lecteur à chauffage classique équipé d'un photomultiplicateur type EMI 9824 devant lequel est placé un filtre ATERVEX Ta-1. II permet de faire une préchauffe avec palier de température réglable fixée pour notre étude à $120^{\circ} \mathrm{C}$ pendant $14 \mathrm{~s}$. La vitesse de préchauffe est de $15^{\circ} \mathrm{C} . \mathrm{s}^{-1}$. La vitesse de chauffe est de $21,3^{\circ} \mathrm{C} . \mathrm{s}^{-1}$. On intègre le nombre de photons émis pendant la chauffe. Pour les réglages, on associe un enregistreur potentiométrique SERVOTRACE SEFRAM type PED : on obtient simultanément le thermogramme correspondant.

- Le lecteur PCL 1 est le prototype d'un lecteur à fours isothermes dans lequel le chargement et le déchargement du matériau RTL se fait automatiquement [10]. II est équipé d'un photomultiplicateur EMI-9825 B à bas bruit de fond devant lequel on a placé un filtre de type MTO 326 dont la bande passante est adaptée au $\mathrm{Li}_{2} \mathrm{~B}_{4} \mathrm{O}_{7}: \mathrm{Cu}$ et au LiF. Le filtre est interchangeable. Un microprocesseur permet de commander le lecteur et de contrôler :

- la durée de l'arrêt sur les fours de préchauffage et de chauffage (12 $\mathrm{s}$ pour les deux fours dans le cas de notre étude) ;

- le délai entre le moment où le matériau arrive sur le four et le début de l'intégration (nul dans le cas de cette étude);

- la durée de l'intégration du signal émis par le matériau lorsqu'il se trouve sur le four de chauffage (12 s dans le cas de notre étude);

- la saisie des informations pour traitement et la sortie des résultats que l'on peut lire sur écran ou imprimer sur papier.

Qu'il s'agisse de l'un ou l'autre lecteur, les matériaux RTL ont toujours été lus dans une atmosphère d'azote $U$ (pureté $99,995 \%$ ) pour éliminer les effets dus à l'oxygène adsorbé et à la triboluminescence. 


\subsection{Les matériaux}

Nous utilisons essentiellement du borate de lithium car il est équivalent au tissu et ne nécessite pas de régénération entre deux utilisations. II peut être soit dopé au manganèse et émettre une lumière de couleur orangée (émission à $600 \mathrm{~nm}$ ), soit au cuivre et émettre une couleur bleutée (émission à $368 \mathrm{~nm}$ ). Suivant nos applications, nous avons été amenés à utiliser des disques de téflon-borate au manganèse TELEDYNE ISOTOPES de type DLB 0.13 (épaisseur $0,13 \mathrm{~mm}$, diamètre $9,5 \mathrm{~mm}$, composition : $95 \%$ téflon - $4,9 \%$ borate - $0,1 \%$ manganèse), de la poudre de borate de lithium dopée au cuivre (proportion de dopant $0,1 \%$ en moles soit $375 \mathrm{ppm})^{*}$.

Les dosimètres solides ont été numérotés et étalonnés individuellement avant utilisation pour prendre en compte une éventuelle différence de sensibilitè. Les échantillons de poudre ont été pesés à l'aide d'une balance METLER type AC 100 (précision : $\pm 0,1 \mathrm{mg}$ ).

\subsection{Les sources d'irradiation}

Elles sont au nombre de trois :

a) un appareil de télécobalthérapie de type ALCYON (GE-CGR MeV) délivrant un débit de dose de l'ordre de $1 \mathrm{~Gy}^{\mathrm{min}}{ }^{-1}$ à $80 \mathrm{~cm}$ de la source ;

b) un accélérateur de type ORION (GE-CGR MeV) délivrant des rayons $X$ de $4 M V$ (indice de qualité $=0,621$ ) avec un débit de dose de lordre de 2 Gy. $\min ^{-1}$ à $100 \mathrm{~cm}$ de la source ;

c) un accélérateur de type SAGITTAIRE (GE-CGR MeV) délivrant à la fois des rayons $X$ de $25 \mathrm{MV}$ et des électrons d'énergies $8,10,13,16,19$, $22,25,28$ ou $32 \mathrm{MeV}$. Le débit de l'accélérateur est réglable de 0,5 Gy à $4 \mathrm{~Gy} \cdot \mathrm{min}^{-1}$ par pas de 0,5 Gy.

Tous ces appareils permettent d'obtenir des champs d'irradiation de dimension supérieure ou égale à $33 \mathrm{~cm} \times 33 \mathrm{~cm}$ autorisant l'irradiation de nombreux échantillons RTL en même temps.

\section{QUE DEVIENNENT LES PROPRIÉTÉS DOSIMÉTRIQUES d'UN MATERIAU QUAND ON CHANGE LE MODE DE CHAUFface?}

Les propriétés dosimétriques dépendent étroitement des conditions de lecture et des traitements subis par le matériau RTL. Cela peut expliquer la diversité des résultats souvent constatée entre des publications traitant pourtant du même matériau. Avant d'entreprendre des mesures systématiques ou des expériences avec un dosimètre thermoluminescent même connu, on aura donc soin de contrôler, voire d'établir si nécessaire, ses principales propriétés dosimétriques en lisant le matériau sur sa propre installation. C'est ce que nous avons fait pour nos deux types de lecteurs et nos deux matériaux.

* Mise au point conjointement par le Service d'instrumentation et de dosimétrie en radioprotection du Commissariat a l'énergie atomique, l'unité de radiophysique de l'hópital Henri-Mondor et le laboratoire de luminescence de Paris VI, grace aux aides $n^{\circ} 82 \mathrm{M}-1042$ a 1044 du Ministère de la recherche et de la technologie [6-7, 13]. 


\subsection{Stabllité de la réponse ou "fading"}

Il est indispensable de s'assurer que les pièges remplis au moment de l'irradiation ne se videront pas avant la lecture par chauffage " fading" thermique), par effet de la lumière ("fading" optique) ou par un moyen quelconque ("fading" anormal).

Pour éviter le "fading" thermique, il faut n'utiliser que les pics de TL "stables" dont la durée de vie à la température ambiante est très grande, donc éliminer les pics de basse température (fig. 1) présentant un "fading" appréciable par phosphorescence à température ambiante en accord avec les lois de la cinétique de TL (McKEEVER, 1985). Pour le borate de lithium, on a le choix entre deux méthodes : l'attente ou le préchauffage. Dans le cas du borate de lithium dopé au manganèse (fig. 1-B), par exemple, attendre $48 \mathrm{~h}$ avant d'effectuer la lecture oc préchauffer le matériau revient au même [3]. Néanmoins, cette méthode a perdu beaucoup de son intérêt depuis que les lecteurs sont munis d'un système de préchauffage ; pour notre étude, nous avons préféré vider les pièges les plus instables par un préchauffage adéquat juste avant la lecture. La recherche des meilleurs paramètres de réglage du préchauffage a été délicate pour le borate dopé au cuivre dont les pics dits "dosimétriques" notés II à IV sont mal séparés du pic instable de basse température noté I (fig. 1-A).

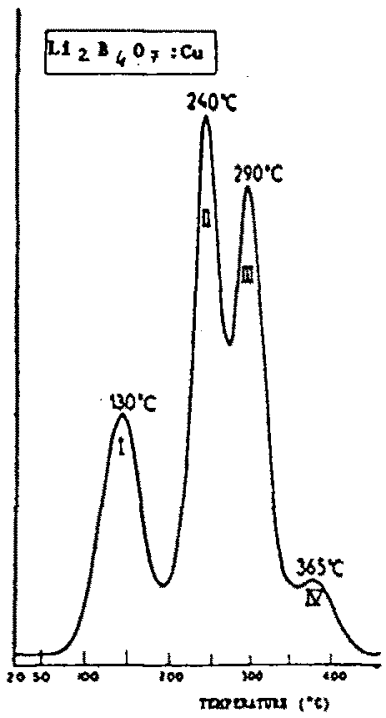

[A]

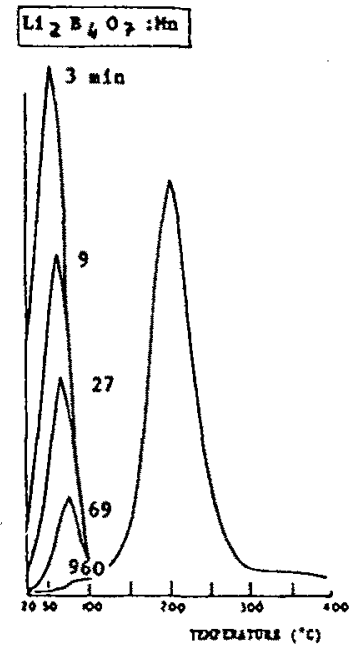

[B]

Fig. 1. - Courbes de thermoluminescence du $\mathrm{Li}_{2} \mathrm{~B}_{4} \mathrm{O}_{7}: \mathrm{Cu}$ avec ses quatre pice princlpaux notés I a IV (A) et du $\mathrm{Li}_{2} \mathrm{~B}_{4} \mathrm{O}_{7}$ : Mn relevées a des délals variables séparant l'irradlation de la lecture, d'apres SCHULMAN et Coll., 1965 [11]. 
Néanmoins, lorsque les conditions de réglage du préchauffage sont optimales, on a obtenu les mêmes courbes de réponse en fonction du délai séparant l'irradiation de la lecture pour un matériau donné, quels que soient le type de lecteur et son mode de chauffage.

Pour éviter le "fading" optique particulièrement important pour certains matériaux, il est indispensable de les manipuler à l'abri de la lumière. On y prêtera particulièrement attention au moment d'effectuer des mesures in vivo : les tubes creux, récipients ou petites enveloppes que l'on pose sur la peau des malades ou que l'on introduit dans les cavités naturelles, devront obligatoirement être opaques.

\subsection{Reproductibilité des mesures}

Elle est étroitement liée à la qualité du matériau, aux caractéristiques du lecteur et, à matériau et lecteur donnés, aux conditions de réglage du cycle de préchauffage et de chauffage. Ce réglage s'est avéré plus critique pour le borate au cuivre qu'il ne l'est pour le borate au manganèse. Cela était prévisible du fait que le pic instable "l" est plus près du pic principal "II" pour le borate au cuivre qu'il ne l'est pour le borate au manganèse (fig. 1). D'autre part, le lecteur SAPHYMO LDT 22, comme la plupart des lecteurs à chauffage traditionnel, ne permet pas d'atteindre la température de $400^{\circ} \mathrm{C}$ nécessaire pour vider tous les pics du borate au cuivre. Néanmoins, on a pu optimiser les conditions de lecture pour les deux types de matériaux et obtenir en routine une reproductibilité meilleure que $\pm 2 \%$ pour les disques DLB 0.13 lus sur le lecteur SAPHYMO LDT 22 (préchauffage pendant $12 \mathrm{~s}$ à $110^{\circ} \mathrm{C}$ et chauffage pendant $7 \mathrm{~s}$ à $\left.264{ }^{\circ} \mathrm{C}\right), \pm 2 \%$ pour le borate au cuivre lu sur le lecteur SAPHYMO LDT 22 (préchauffage $14 \mathrm{~s}$ à $120^{\circ} \mathrm{C}$ et chauffage $10 \mathrm{~s}$ à $268^{\circ} \mathrm{C}$ ) et \pm $1,5 \%$ pour la poudre de $\mathrm{Li}_{2} \mathrm{~B}_{4} \mathrm{O}_{7}$ : Cu lue sur le lecteur automatique PCL1 (préchauffage $12 \mathrm{~s}$ à $173^{\circ} \mathrm{C}$ et chauffage à $12 \mathrm{~s}$ à $475^{\circ} \mathrm{C}$ ).

Les températures indiquées ci-dessus ne sont données qu'à titre indicatif car l'affichage peut varier suivant le type de coupelle ou de plaquette utilisées. D'autre part, la reproductibilité des mesures effectuées avec la poudre est celle obtenue en pesant soigneusement les échantillons de poudre avant toute mesure.

\subsection{Variation de la réponse en fonction de la dose}

Les conditions de réglage du chauffage et du préchauffage ayant été optimisées pour les deux types de lecteurs et les deux types de matériaux, on a établi la courbe de réponse en fonction de la dose de ces derniers en irradiant des échantillons à doses croissantes dans un faisceau de cobalt 60 . Les résultats (fig. 2) montrent que les réponses obtenues ne dépendent alors que du matériau et non du type de lecteur sous réserve qu'elles solent normalisées à 1 pour une même dose. 


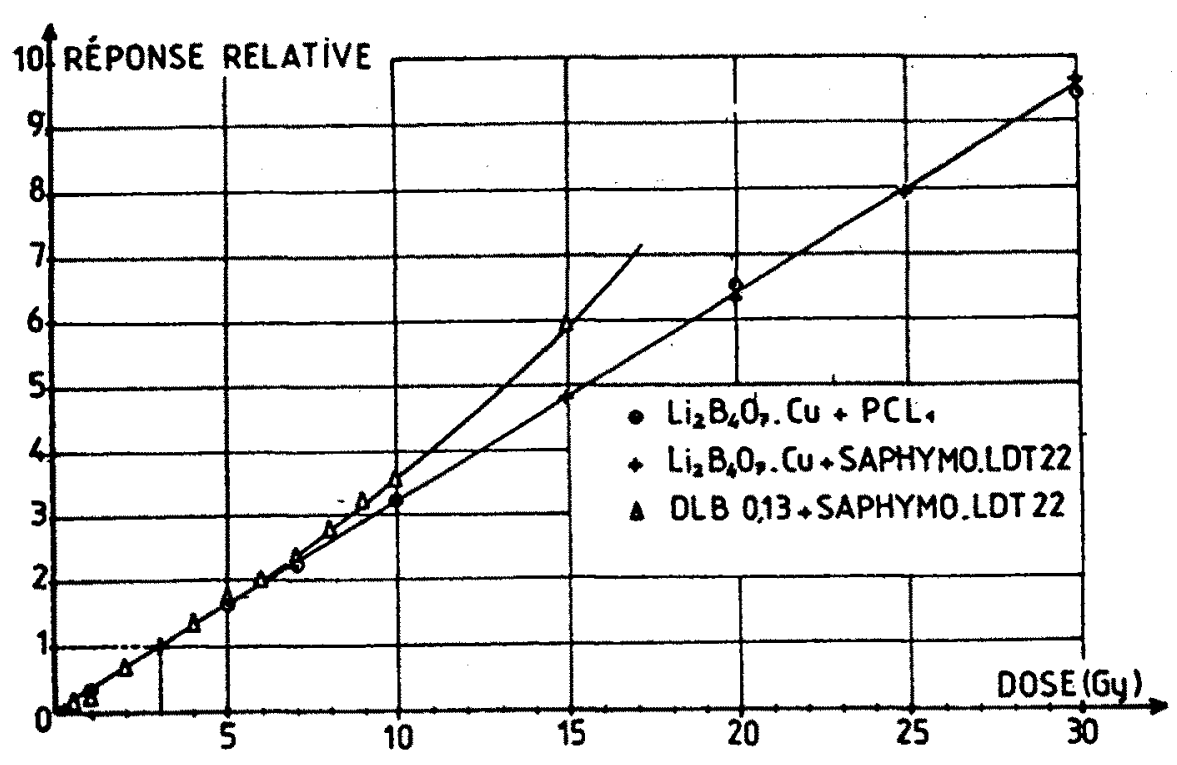

Fig. 2. - Variation de réponse des borates en fonction de la dose.

\subsection{Variation de la réponse en fonction de la masse}

Lorsque l'on utilise des matériaux RTL se présentant sous forme pulvérulente, on doit lire des quantités de poudre adaptées à la taille de l'élément chauffant servant à contenir cette dernière. En particulier, il faut que la poudre recouvre complètement le fond de la coupelle, qu'elle soit répartie le plus régulièrement possible et que son épaisseur ne soit pas trop importante de façon à ce qu'il n'y ait pas de gradient thermique à l'intérieur de l'échantillon.

Ces conditions étant remplies, un certain nombre de matériaux RTL ont une réponse proportionnelle à la masse de poudre. II n'en est pas de même pour le $\mathrm{Li}_{2} \mathrm{~B}_{4} \mathrm{O}_{7}: \mathrm{Cu}$. Ceci est dû à un phénomène d'auto-absorption de la lumière émise dans le matériau lui-même sans doute lié à la longueur d'onde d'émission. Nous avons, en effet, montré qu'à masse égale et à conditions de lecture identiques la réponse du matériau augmente quand la poudre est répartie sur une coupelle de diamètre de plus en plus grand.

II en résulte que la variation de la réponse du $\mathrm{Li}_{2} \mathrm{~B}_{4} \mathrm{O}_{7}: \mathrm{Cu}$ en fonction de la masse de poudre lue dépend beaucoup du type de lecteur et, pour un lecteur donné, des conditions de chauffage (fig. 3). L'examen de cette figure montre que, si le lecteur autorise un chauffage à très haute température, on peut trouver des conditions de réglage du chauffage telles que la quantité de lumière émise ne dépende que très peu dé la masse 


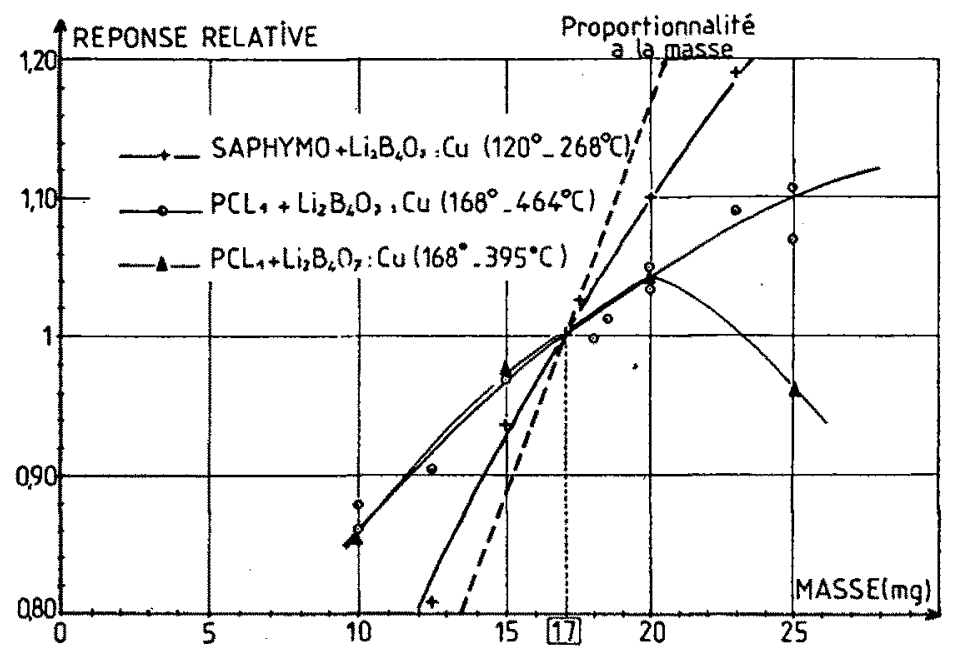

Fig. 3. - Varlation de roponse du $\mathrm{L}_{2} \mathrm{~B}_{4} \mathrm{O}_{7}$ : $\mathrm{Cu}$ en fonction de la masae (les echantlllons de poudre sont lus dans une coupelle de $5 \mathrm{~mm}$ de dlametre).

de poudre contenue dans la coupelle. On observe, par exemple, une variation de réponse $\pm 2 \%$ pour des masses de poudre variant de 15 à $18,5 \mathrm{mg}$ étalée dans une coupelle de $5 \mathrm{~mm}$ de diamètre, quand on pose cette dernière $12 \mathrm{~s}$ sur un four isotherme à $395^{\circ} \mathrm{C}$ (lecteur automatique PCL1). Dans ces conditions, il devient inutile de peser soigneusement les échantillons avant toute mesure, et l'on peut se contenter en routine d'utiliser un doseur (fig. 4) qui fournit, en général, une précision meilleure que le milligramme dans le domaine des masses qui nous intéresse en pratique. Le gain de temps est alors considérable.

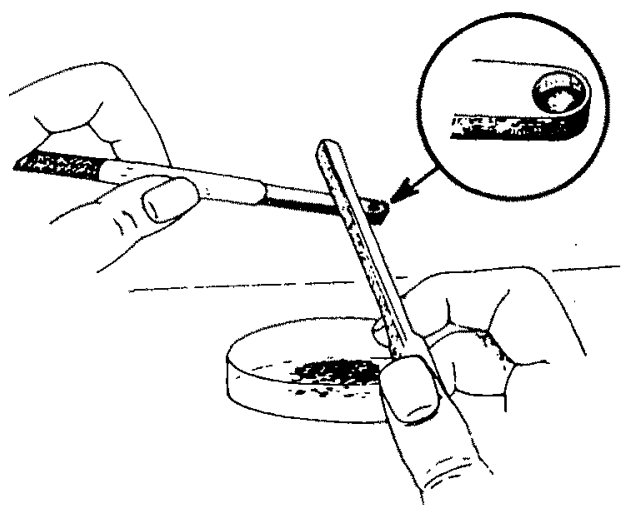

Fig. 4. - Exemple de doseur. 
Lorsqu'il n'est pas possible d'optimiser le chauffage, on doit procéder comme pour les matériaux dont la réponse TL est proportionnelle à la masse, c'est-à-dire utiliser des échantillons de masse égale ou appliquer des facteurs de correction appropriés établis dans ses propres conditions expérimentales.

\subsection{Varlation de la réponse en fonction de l'énergle}

La dosimétrie appliquée au domaine médical ou à la radioprotection des personnes concerne la détermination de la dose absorbée dans les milieux biologiques assimilables à l'eau en première approximation. Parmi les différents dosimètres disponibles sur le marché, le borate de lithium est l'un de ceux dont la réponse en fonction de l'énergie est la plus proche de l'eau, quel que soit le type de rayonnement considéré, à condition que la proportion du dopant soit très faible.

\subsubsection{Falsceaux de photons de haute énergle}

La variation du rapport des coefficients massiques d'absorption calculés dans l'eau et dans le borate de. lithium en fonction de l'énergie des photons incidents est présentée sur la figure 5. Théoriquement, connaissant les coefficients massiques d'absorption des photons dans l'eau et le matériau thermoluminescent, il est possible de calculer la dose absorbée dans l'eau à partir de la dose absorbée dans le matériau thermoluminescent. Mais le calcul devient difficile pour les photons de haute énergie. En effet, les électrons secondaires pris en compte dans la réponse du dosimètre proviennent à la fois du milieu équivalent au tissu environnant et du dosimètre lui-même, notamment en raison de la taille du dosimètre et du parcours des électrons secondaires. Aussi, les études théoriques qui permettent de passer de la dose dans un dosimètre solide à la dose dans le milieu environnant sontelles relativement complexes et quelquefois contradictoires [1-2].

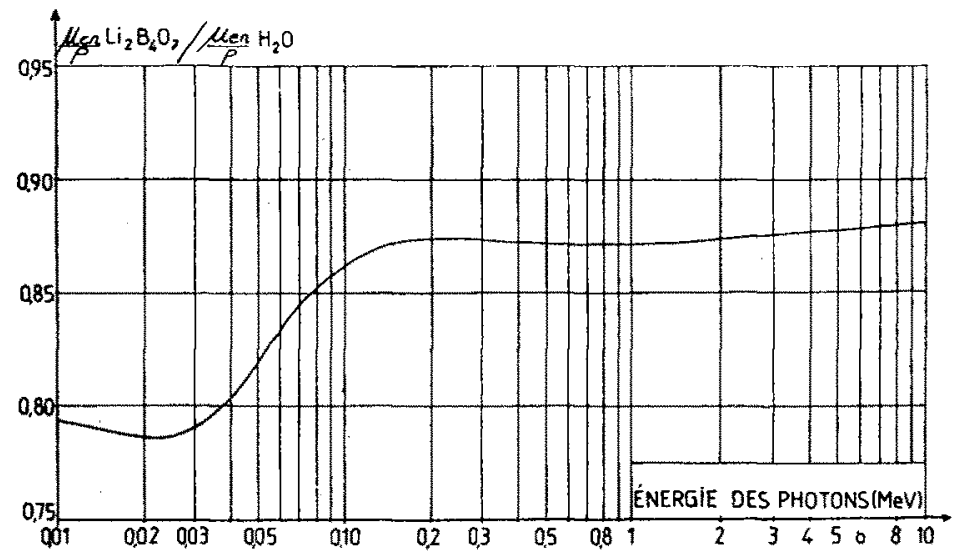

Fig. 5. - Verlation du rapport des coeffleients massiques d'absorption dans le borate de lithium et dans l'eau en fonction de l'énergie des photons incldents calculé a partir de [8]. 
Nous avons préféré comparer directement la réponse des borates de lithium à celle d'une chambre d'ionisation cylindrique NUCLEAR ENTERPRISES 2503/B connectée à un dosimètre IONEX type 2500 (fig. 6).

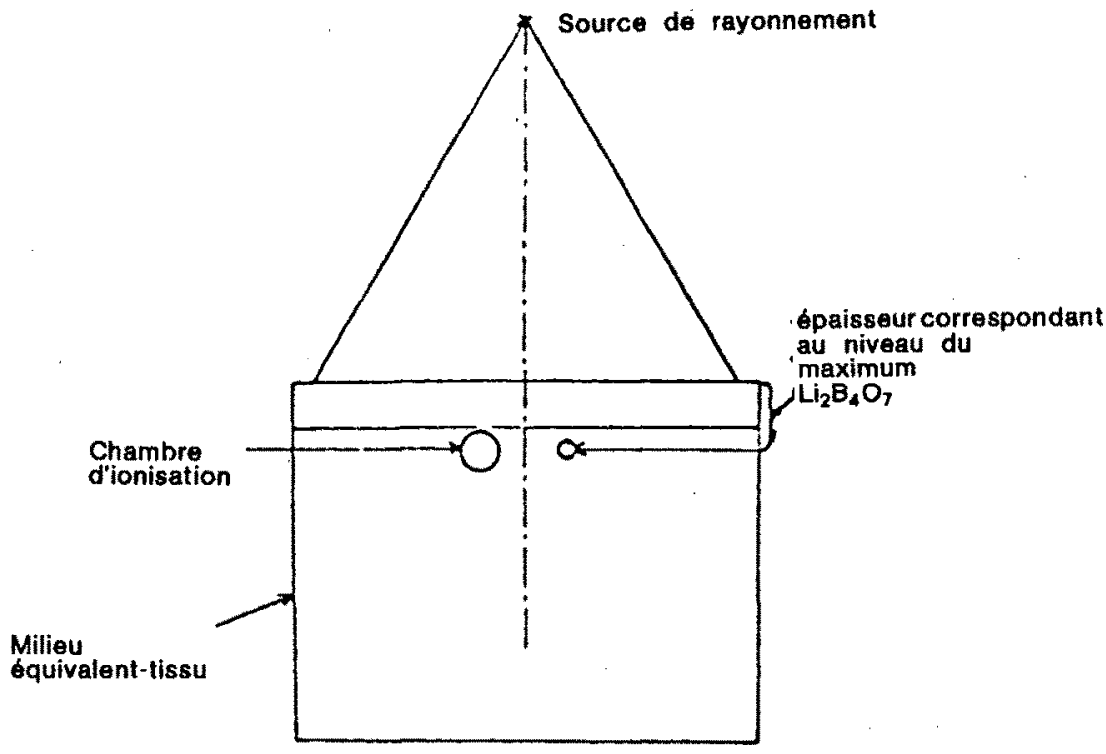

Fig. 6. - Montage expérimental utilisé pour l'étalonnage du matériau en fonction de l'ónergie.

La comparaison des rèponses des deux détecteurs permet de déduire le coefficient $T_{u}$, borate, $\mathrm{Rx}$ permettant de passer de la réponse du borate dans un faisceau de cobalt 60 (lecture $\times T_{u}$, borate, ${ }^{60} \mathrm{Co}_{0}$ ) à sa réponse dans un faisceau de rayons $X$ d'énergie $E$. On peut alors calculer la dose absorbée dans le milieu équivalent à l'eau à l'aide de la relation suivante :

Dose à l'énergie $E=$ lecture $\times T_{u \text {, borate, }}{ }^{60} c_{c o} \times T_{u \text {, borate, } \mathrm{RX}}$

Le coefficient obtenu en irradiant les disques de borate de lithium dopé au manganèse (DLB 0.13 ) avec des rayons $X$ de $25 \mathrm{MV}$ et en les lisant sur le lecteur à chauffage traditionnel SAPHYMO LDT 22 est égal à $0,98 \pm 0,02$ (CHAVAUDRA, 1976).

Quant aux mesures effectuées avec de la poudre de $\mathrm{Li}_{2} \mathrm{~B}_{4} \mathrm{O}_{7}$ : Cu irradiée avec des rayons $X$ de $25 \mathrm{MV}$, elles donnent des coefficients égaux à :

- $T_{u \text {, borate, } 25 \mathrm{MV}}=1,03 \pm 0,015$ quand la poudre est lue sur le lecteur SAPHYMO LDT 22 ,

- $T_{U, \text { borate, } 25 \mathrm{mV}}=0,99 \pm 0,015$ quand la poudre est lue sur le lecteur à chauffage rapide PCL1. 
A conditions expérimentales identiques, on observe donc une réponse en fonction de l'énergie différente suivant le mode de chauffage. II est à noter que le coefficient correspondant à l'énergie moyenne du faisceau que l'on aurait déduit de la courbe théorique de la figure 5 est 0,99 .

\subsubsection{Les falsceaux d'électrons de haute énergie}

Dans ce cas, la variation de réponse en fonction de l'énergie est liée à la variation du rapport des pouvoirs de ralentissement des électrons dans le borate et dans l'eau en fonction de l'énergie des électrons incidents. Une détermination expérimentale analogue à celle utilisée pour les photons nous a permis d'obtenir différentes valeurs de $T_{u \text {, borate, }} \mathrm{e}^{-}$en fonction de l'énergie moyenne des électrons au niveau du point de mesure calculée conformément aux indications du rapport de l'AIEA [4].

Les disques de borate au manganèse DLB 0.13 lus sur le SAPHYMO LDT 22 correspondent à des coefficients variant de 1,07 $\pm 0,02$ à 1,04 $\pm 0,02$ dans le domaine de 5 à $25 \mathrm{MeV}$ [3].

Quant à la poudre de borate au cuivre, sa réponse est différente suivant le mode de chauffage du matériau. On constate, en effet, une variation inférieure à $2 \%$ entre 3 et $20 \mathrm{MeV}$, quand le matériau est lu sur le lecteur SAPHYMO LDT 22, et de l'ordre de $5 \%$, lorsqu'il est lu sur le lecteur PCL1. On remarque que la variation calculée à partir du rapport des pouvoirs de ralentissement des électrons est de $1,5 \%$ dans ce domaine d'énergie.

\section{CONCLUSION}

Ce travail montre que les propriétés dosimétriques d'un matériau thermoluminescent donné dépendent, pour un certain nombre d'entre elles, des conditions de chauffage du matériau : elles doivent donc être déterminées par chaque utilisateur dans ses propres conditions de lecture.

II montre aussi qu'un choix judicieux du matériau associé à un mode de chauffage adéquat permet parfois un gain de temps considérable, sous réserve que les conditions de lecture soient correctement optimisées. Ainsi, pour effectuer la dosimétrie in vivo des malades irradiés in toto avant greffe de moelle osseuse, le fait de passer en routine des disques de borate de lithium dopé au manganése lus sur un lecteur à chauffage traditionnel à la poudre de borate de lithium dópée au cuivre lue sur un lecteur automatique à chauffage rapide, nous a fait gagner un facteur 3 sur le temps total (incluant la préparation des dosimètres) a consacrer par malade pour ce type d'irradiation.

Enfin, cette étude limitée ici au seul borate de lithium est actuellement étendue à d'autres types de matériaux. 


\section{BIBLIOGRAPHIE}

[1] ALMOND P., McGRAY K. - The energy response of $\mathrm{LiF}_{,} \mathrm{CaF}_{2}$ and $\mathrm{Li}_{2} \mathrm{~B}_{4} \mathrm{O}_{7}: \mathrm{Mn}$ to high energy radiations. Phys. Med. Biol, 1970, 15, 335-342.

[2] BURLIN T.E. - The energy response of $\mathrm{LiF}, \mathrm{CaF}_{2}$ and $\mathrm{Li}_{2} \mathrm{~B}_{4} \mathrm{O}_{7}$ to high energy radiations. Phys. Med. Biol., 1970, 4, 558-560.

[3] CHAVAUDRA J., MARINELLO G., BRULE A.M. NGUYEN J. - Utilisation pratique du borate de lithium en dosimétrie par thermoluminescence. J. Radiol. Electrol., 1976, 57, 435-445.

[4] INTERNATIONAL ATOMIC ENERGY AGENCY (AIEA). - Absorbed dose determination in photon and electron beams. Vienne : AIEA, 1987.

[5] INTERNATIONAL COMMISSION ON RADIATION UNITS AND MEASUREMENTS (ICRU). - Electron with initial energies between 1 and $50 \mathrm{MeV}$ (ICRU report 35) Washington D.C. : ICRU, 1986.

[6] LORRAIN S., MARINELLO G, VISOCEKAS R. - Développement d'un matériau thermoluminescent pour la dosimétrie des radiations ionisantes, le borate de lithium activé au $\mathrm{Cu}$. Compte rendu de fin de recherche, aides du ministère de la recherche et de la technologie (Contrats $n^{\circ} 82-M-1042$ à 1044), 1984.

[7] LORRAIN S., DAVID J.P., VISOCEKAS R., MARINELLO G. - A study of new preparations of radiothermoluminescent lithium borates with various activators. Radiat. Prot. Dosim., 1986, 17, 385-392.

[8] LOS ALAMOS SCIENTIFIC LABORATORY. - Photon cross sections from 0.001 to $100 \mathrm{MeV}$ for elements 1 through 100. Rapport LAMS.

[9] MCKEEVER S.W.S. - Thermoluminescence of solids. Cambridge : University press, 1985.

[10] PORTAL G. - Lecteur automatique d'échantillons frittés ou poudrés thermoluminescents. Communication personnelle, 1983.

[11] SCHULMAN J.H., KIRK R.D., WEST E.J. - Use of lithium borate for thermoluminescence dosimetry - In : Proceedings of the International Conference on luminescence dosimetry, Stanford, 1965.

[12] THOMSON J.J., ZIEMER P.L. - Energy response of lithium borate thermoluminescent dosimeters. Health Phys., 1972, 22, 399-401.

[13] VISOCEKAS R., LORRAIN S., MARINELLO G. - Evaluation of a preparation of $\mathrm{Li}_{2} \mathrm{~B}_{4} \mathrm{O}_{7}$ : Cu for thermoluminescence dosimetry. Nucl. Sci. J., 1985, 22,61-66. 